\title{
CONTROL PREDICTIVO PARA LA OPERACIÓN EFICIENTE DE UNA PLANTA FORMADA POR UN SISTEMA DE DESALACIÓN SOLAR Y UN INVERNADERO
}

\author{
Juan D. Gil ${ }^{1}$, Lidia Roca ${ }^{2}$, Manuel Berenguel ${ }^{1}$, Alba Ruiz-Aguirre ${ }^{1}$, Guillermo Zaragoza ${ }^{2}$, Antonio Giménez ${ }^{1}$ \\ ${ }^{1}$ Centro Mixto CIESOL, ceiA3, Universidad de Almería. \\ \{juandiego.gil,beren,ara399,agimfer\}@ual.es \\ ${ }^{2}$ Centro Mixto CIESOL, CIEMAT-Plataforma Solar de Almería. \\ \{lidia.roca,guillermo.zaragoza\}@psa.es
}

\section{Resumen}

El déficit de agua en la zona del Mediterráneo afecta de forma directa a la agricultura. Una de las posibles soluciones consiste en suministrar el agua requerida por los cultivos mediante procesos de desalación térmica. En este trabajo, se propone un esquema de control predictivo no lineal (NMPC, Nonlinear Model Predictive Control) para gestionar el reparto del caudal de alimentación entre los diferentes módulos de destilación que componen la planta de desalación. El objetivo del problema de optimización consistirá en obtener una operación óptima en términos de eficiencia térmica de la instalación, al mismo tiempo que se satisfaga la demanda de agua del invernadero. Los resultados preliminares en simulación ponen de manifiesto la eficacia de la técnica desarrollada.

Palabras clave: Control de procesos, Control predictivo no lineal, Eficiencia térmica, Destilación por membranas.

\section{INTRODUCCIÓN}

Hoy en día, dos de los grandes retos a los que se debe enfrentar la humanidad son el cambio climático y la escasez de agua. Por este motivo, la gestión racional de los recursos hídricos y la promoción del uso de energías renovables, se han convertido en temas de vital importancia en el desarrollo mundial. En este contexto, la utilización de sistemas de desalación térmica alimentados con energía solar, representa una solución adecuada para el abastecimiento de agua en explotaciones agrícolas, especialmente en zonas áridas o semi-áridas con escasez de agua y grandes superficies de cultivos, como es el caso de la cuenca del Mediterráneo.

Uno de los sistemas de cultivo más extendidos en el sudeste de España son los invernaderos. La principal ventaja de este tipo de sistemas radica en que la productividad puede ser controlada a través de la correcta manipulación de las variables medioambientales, y la cantidad de agua y fertilizantes utilizados para el riego. De este modo, el uso de invernaderos se ha extendido de forma rápida, afectando severamente a las reservas de agua. Este hecho es especialmente notable en la cuenca del Mediterráneo [10, 4]. Consecuentemente, la sostenibilidad del sistema de invernaderos en esta zona está comprometida, y por tanto, la búsqueda de fuentes de agua alternativas se ha convertido en uno de los objetivos prioritarios.

Los procesos de desalación impulsados con energía solar pueden ser una de las soluciones más adecuadas para combatir el déficit hídrico en zonas con buena disponibilidad de irradiancia solar y acceso a agua de mar, como es el caso del área del Mediterráneo. La destilación por membranas con apoyo de energía solar (SMD, Solar Membrane Distillation), es una tecnología que está siendo investigada para el desarrollo de pequeñas plantas autosuficientes de desalación [19]. Esta tecnología destaca por su baja temperatura de operación, la cual, permite que sea fácilmente combinable con tecnologías solares. La destilación por membranas (MD, Membrane Distillation) es un proceso impulsado térmicamente, en el que la fuerza impulsora es la diferencia de presión de vapor entre ambos lados de una membrana hidrófoba y microporosa. Los sistemas MD suelen ser clasificados dependiendo del lugar donde se produzca la condensación del permeado [1]. La configuración empleada en este trabajo es la Destilación por Membranas con Canal de Permeado (PGMD, Permeate Gap MD), en la cual, la condensación tiene lugar dentro del propio módulo. A pesar de su amplio rango de buenas características, la tecnología SMD tiene una serie de inconvenientes técnicos que han impedido hasta el momento que se comercialice a nivel industrial [7]. Uno de lo impedimentos más significativos es la baja eficiencia térmica del proceso.

En la mayoría de los trabajos que se pueden encontrar hasta el momento en la literatura, se proponen algoritmos de optimización y control para maximizar la producción de destilado [14], o bien para maximizar la eficiencia térmica de la planta $[2,8,15]$. Sin embargo, hay muy pocos trabajos que tratan de optimizar la operación de una planta de destilación con el objetivo de satisfacer una determinada demanda de agua. En este sentido, en [16] se propone un algoritmo de control predicti- 
vo para la operación de una planta de destilación multi-efecto, con el objetivo de satisfacer la demanda de agua diaria variable de un invernadero.

En este trabajo, se presenta un algoritmo de control predictivo para la operación óptima, en términos de eficiencia térmica, de un sistema formado por una planta SMD y un invernadero. El reto del presente trabajo consiste en maximizar la eficiencia térmica de la operación al mismo tiempo que se satisfaga la demanda de agua del invernadero, objetivos que requieren condiciones de operación contrarias. Para ello, se propone un algoritmo de control basado en un enfoque práctico del NMPC (PNMPC, Practical Nonlinear Model Predictive Control), con el que se pretende realizar una gestión óptima del caudal de alimentación para los diferentes módulos MD que componen la planta de desalación. Además, el algoritmo se complementa con el uso de una técnica de suavizado exponencial doble (DES, Double Exponential Smoothing), para la predicción de la radiación [12]. Los resultados preliminares obtenidos en simulación se comparan con una operación típica sin PNMPC, con el objetivo de analizar las ventajas del sistema de control propuesto.

\section{DESCRIPCIÓN DEL SISTEMA}

En la Fig. 3 se presenta el diagrama esquemático de la planta estudiada. En esta planta, se debe gestionar la interconexión de dos instalaciones: un invernadero y una instalación SMD. El invernadero demanda agua para el riego diario de los cultivos, mientras que la desaladora produce destilado en módulos MD. Además, se dispone de un tanque de almacenamiento de destilado de $3 \mathrm{~m}^{3}$. Se debe hacer notar que la instalación considerada es un caso de estudio en simulación, basado en las dos instalaciones reales que se describen en las siguientes subsecciones.

\subsection{Planta SMD}

La planta piloto SMD (ver Fig. 1) de la Plataforma Solar de Almería (PSA, www.psa.es), es una de las pocas instalaciones MD totalmente descrita en la literatura [19]. En ella, la energía térmica necesaria para el proceso de destilado la proporciona un campo solar formado por dos filas de cinco captadores planos cada una. La salida del campo solar está conectada a un tanque de almacenamiento térmicamente aislado (1500 L), que se emplea como buffer o almacenamiento energético. Por último, los módulos se conectan al tanque a través de un intercambiador de calor, el cual se utiliza para calentar la solución de alimentación con el fluido proveniente del sistema de generación de energía. Todas las variables registradas en esta planta se muestrean cada segundo.

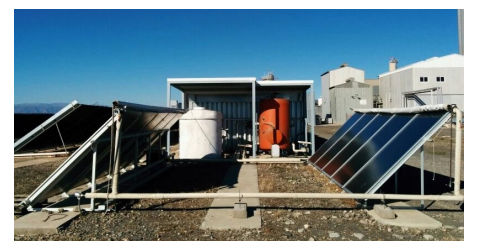

Figura 1: Instalación piloto SMD en la PSA.

\subsection{Invernadero}

El invernadero empleado como referencia en este trabajo (ver Fig. 2) se encuentra en la Estación Experimental de Investigación de la fundación Cajamar en El Ejido, provincia de Almería. El invernadero tiene una superficie de $800 \mathrm{~m}^{2}$ con un área de cultivo de $616 \mathrm{~m}^{2}$. El material de la cubierta es polietileno, y cuenta con un sistema de ventilación automática con ventanas laterales en las paredes norte y sur. El cultivo crece en sacos de fibra de coco con seis plantas cada uno y tres goteros. En [16] fue presentada una descripción más detallada del invernadero. Las variables registradas en esta planta se muestrean cada 30 segundos.

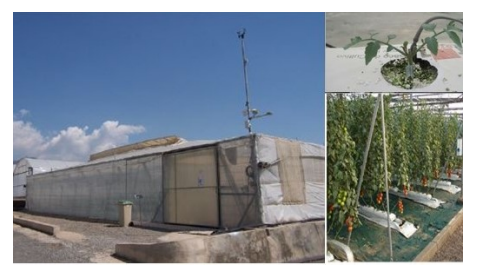

Figura 2: Instalaciones del invernadero. Desde la izquierda hacia la derecha y desde arriba hacia abajo: invernadero, pipeta de riego y líneas de cultivo de tomates.

\section{Modelado del sistema}

Para la prueba del sistema de control propuesto, es necesario desarrollar un modelo que represente con exactitud el comportamiento de ambos sistemas.

\subsection{Modelo de la planta SMD}

El modelo de la planta SMD se puede dividir en dos grandes componentes unidos a través de un intercambiador de calor: el sistema de generación de energía, formado por el campo solar y el tanque de almacenamiento, y la unidad de desalación MD. El modelo del sistema de sistema de generación de energía térmica ya ha sido presentado en $[5,6]$. Para este trabajo, con el objetivo de simplificar la simulación, en lugar de utilizar el modelo 


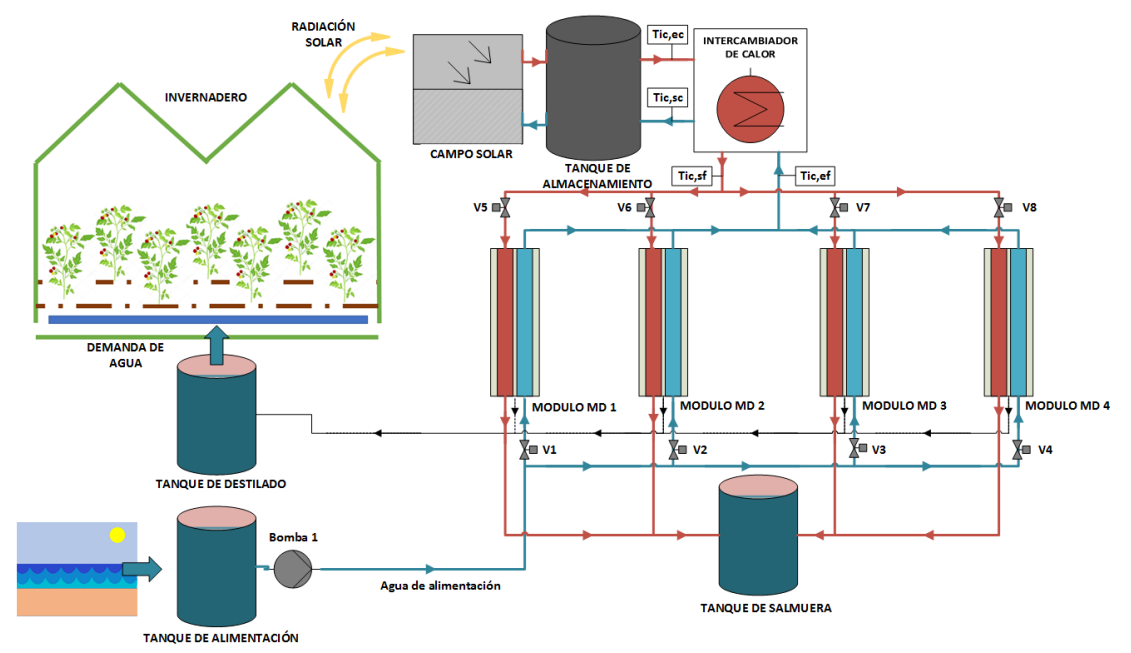

Figura 3: Diagrama esquemático de la instalación simulada como caso de estudio.

del sistema de generación de energía, se utilizarán perfiles de temperatura a la entrada del intercambiador de calor por la parte caliente, obtenidos mediante simulaciones del modelo del sistema de generación de energía, realizadas en días con condiciones meteorológicas similares a los utilizadas para las pruebas en simulación de este trabajo, y utilizando la estrategia de operación presentada en [5]. Por otra parte, el intercambiador de calor ha sido modelado mediante un modelo estático basado en primeros principios, como el presentado en [3]:

$$
\begin{gathered}
\mathrm{T}_{i c, s c-m}=\mathrm{T}_{i c, e c}-\eta_{1} \cdot\left(\mathrm{T}_{i c, e c}-\mathrm{T}_{i c, e f}\right),(1) \\
\mathrm{T}_{i c, s f-m}=\mathrm{T}_{i c, e f}+\eta_{2} \cdot\left(\mathrm{T}_{i c, e c}-\mathrm{T}_{i c, s c-m}\right),(2)
\end{gathered}
$$

donde:

$$
\begin{gathered}
\eta_{1}=\frac{1-e^{\theta_{i c}}}{1-\frac{\dot{m}_{1} \cdot c_{p, 1}}{\dot{m}_{2} \cdot c_{p, 2}} e^{\theta_{i c}}}, \\
\eta_{2}=\frac{\dot{m}_{1} \cdot c_{p, 1}}{\dot{m}_{2} \cdot c_{p, 2}}, \\
\theta_{i c}=\alpha_{i c} \cdot A_{i c} \cdot\left(\frac{1}{\dot{m}_{1} \cdot c_{p, 1}}-\frac{1}{\dot{m}_{2} \cdot c_{p, 2}}\right),
\end{gathered}
$$

$\mathrm{T}_{i c, s c-m}$ es la temperatura de salida estimada por el modelo por la parte caliente del intercambiador, $\mathrm{T}_{i c, e c}$ es la temperatura de entrada por la parte caliente del intercambiador, $\mathrm{T}_{i c, \text { ef }}$ es la temperatura de entrada por la parte fría del intercambiador y $\mathrm{T}_{i c, s f-m}$ es la temperatura de salida estimada por el modelo en la parte fría del intercambiador. Las unidades de la temperatura son ${ }^{\circ} \mathrm{C}$. Por otra parte, $\dot{m}_{1}$ y $\dot{m}_{2}$ son el flujo másico en $\mathrm{kg} / \mathrm{s}$ de la parte caliente y fría del intercambiador respectivamente, mientras que $\mathrm{c}_{p, 1}$ y $\mathrm{c}_{p, 2}$ son la capacidad calorífica específica $\left(\mathrm{J} / \mathrm{kg} \cdot{ }^{\circ} \mathrm{C}\right)$ del agua desmineralizada y del agua de mar. Por último, $\alpha_{i c}$ es el coeficiente de transferencia de calor del intercambiador y $\mathrm{A}_{i c}$ es la superficie de intercambio del intercambiador. Para este trabajo se han considerado que los valores de estos parámetros son $689.30 \mathrm{~W} /{ }^{\circ} \mathrm{C} \cdot \mathrm{m}^{2} \mathrm{y}$ $3.15 \mathrm{~m}^{2}$ respectivamente.

Para el modelado del módulo MD, se han llevado a cabo campañas experimentales siguiendo el procedimiento descrito en [18], con el fin de obtener modelos estáticos de la producción de destilado (D) y del $\Delta \mathrm{T}$, que es la diferencia entre la temperatura de salida del canal de condensación de cada módulo (canal azul en la Fig. 3) y la temperatura de entrada al canal de evaporación (canal rojo en la Fig. 3). Las entradas del modelo y su rango de variación son: i) caudal de alimentación (Q), 400-600 L/h, ii) $\mathrm{T}_{i c, s f}$, entre $60-80{ }^{\circ} \mathrm{C} \mathrm{y}$ iii) temperatura del tanque de alimentación $\left(\mathrm{T}_{t a}\right)$, 20-30 ${ }^{\circ} \mathrm{C}$. Así, las ecuaciones estáticas que caracterizan el módulo MD son:

$$
\begin{aligned}
\mathrm{D} & =10 \cdot\left(-1,088+0,024 \cdot \mathrm{T}_{i c, s f}-0,018 \cdot \mathrm{T}_{t a}\right. \\
& \left.-0,001 \cdot \mathrm{Q}+0,00006 \cdot \mathrm{T}_{i c, s f} \cdot \mathrm{Q}\right), \\
\triangle \mathrm{T} & =-0,201875+0,1385 \cdot \mathrm{T}_{i c, s f}-0,158 \cdot \mathrm{T}_{t a} \\
& +0,0019 \cdot \mathrm{Q},
\end{aligned}
$$

donde $\mathrm{D}$ es la producción de destilado en L/h. Las unidades para la temperatura son ${ }^{\circ} \mathrm{C}$ y para el caudal L/h. Por otra parte, el caudal de alimentación de cada módulo se controla mediante las válvulas de entrada (V1, V2, V3 y V4 en Fig. 3), las cuales varían su apertura entre 0 y 1 . Además, durante la operación, V1 y V5, V2 y V6, V3 y V7, y V4 y V8, se abren o cierran al mismo valor, con el objetivo de obtener el mismo reparto de caudales en los dos canales de entrada de cada módulo. Por último, la bomba 1 suministra caudales entre 1600 y $2000 \mathrm{~L} / \mathrm{h}$. 


\subsection{Modelo del invernadero}

En términos generales, la temperatura del aire del invernadero $\left(T_{i n v}\right)$ se puede modelar usando el siguiente balance de energía [17]:

$$
c_{p, a} \rho_{a} \frac{V_{g}}{A_{s s}} \frac{d T_{i n v}}{d t}=q_{c f, c}+q_{c f, s}-q_{v e n}-q_{t r, c},
$$

donde $c_{p, a}$ es la capacidad térmica del aire $\left(\mathrm{J} / \mathrm{kg}^{o} \mathrm{C}\right), \rho_{a}$ es la densidad del aire $\left(\mathrm{kg} / \mathrm{m}^{3}\right), V_{g}$ es el volumen del invernadero $\left(\mathrm{m}^{3}\right)$ y $A_{s s}$ es la superficie del suelo $\left(\mathrm{m}^{2}\right), q_{c f, c}$ es el flujo convectivo $\left(\mathrm{W} / \mathrm{m}^{2}\right)$ con la cubierta, $q_{c f, s}$ es el flujo convectivo $\left(\mathrm{W} / \mathrm{m}^{2}\right)$ con la superficie del suelo, $q_{v e n}\left(\mathrm{~W} / \mathrm{m}^{2}\right)$ representa las pérdidas de calor provocadas por la ventilación natural, y $q_{t r, c}\left(\mathrm{~W} / \mathrm{m}^{2}\right)$ es el calor latente de transpiración del cultivo.

Por otro lado, la humedad absoluta en el interior del invernadero $\left(H_{i n v}\right)$ se puede calcular en base al balance de masa de vapor de agua [17]:

$$
\begin{aligned}
\rho_{a} \frac{V_{a}}{A_{s s}} \frac{d H_{i n v}}{d t} & =\dot{M}_{t r, c}+\dot{M}_{e v p, s} \\
& -\dot{M}_{c o n d, c}-\dot{M}_{v e n t}-\dot{M}_{p},
\end{aligned}
$$

donde $\dot{M}_{t r, c}\left(\mathrm{~kg} / \mathrm{s} \cdot \mathrm{m}^{2}\right)$ es el flujo de transpiración del cultivo, $\dot{M}_{e v p, s}\left(\mathrm{~kg} / \mathrm{s} \cdot \mathrm{m}^{2}\right)$ es la evaporación del suelo, $\dot{M}_{\text {cond,c }}\left(\mathrm{kg} / \mathrm{s} \cdot \mathrm{m}^{2}\right)$ es el flujo de condensado de la cubierta, $\dot{M}_{\text {vent }}\left(\mathrm{kg} / \mathrm{s} \cdot \mathrm{m}^{2}\right)$ es el flujo de salida de la ventilación natural, y $\dot{M}_{p}$ son las pérdidas de humedad por la infiltración.

Con el objetivo de escalar el consumo de agua a los niveles de producción de la planta de desalación, se han utilizado cinco invernaderos con las características descritas en la Sección 2.2.

\section{Análisis de la operación óptima del módulo MD}

Para evaluar la eficiencia térmica de la planta de desalación se pueden utilizar diferentes métricas. En este caso, se utilizará un índice que mide la cantidad de energía térmica necesaria para producir una unidad de destilado (STEC, Specific Thermal Energy Consumption):

$$
\operatorname{STEC}\left[\mathrm{kWh} / \mathrm{m}^{3}\right]=\frac{\mathrm{Q} \cdot \rho \cdot c_{p} \cdot \Delta \mathrm{T}}{c_{f} \cdot \mathrm{D}},
$$

donde $\rho$ es la densidad del agua $\left(\mathrm{kg} / \mathrm{m}^{3}\right), c_{p}$ es la capacidad calorífica del agua $\left(\mathrm{J} / \mathrm{kg} \cdot{ }^{\circ} \mathrm{C}\right)$ y $c_{f}$ es un factor de conversión $\left(3.6 \cdot 10^{6} \mathrm{~J} / \mathrm{kWh}\right)$.

Los valores de eficiencia térmica máxima se consiguen con diferentes condiciones de operación, de- pendiendo del tipo de módulo MD y de la configuración empleada. Para analizar el comportamiento del módulo PGMD empleado en este trabajo se han realizado gráficas $3 \mathrm{D}$, en las cuales se han representado el STEC y la producción de destilado en función de las dos variables principales que influyen en su comportamiento: el caudal de alimentación (Q) y la temperatura a la salida del intercambiador de calor por la parte fría $\left(\mathrm{T}_{i c, s f}\right)$.

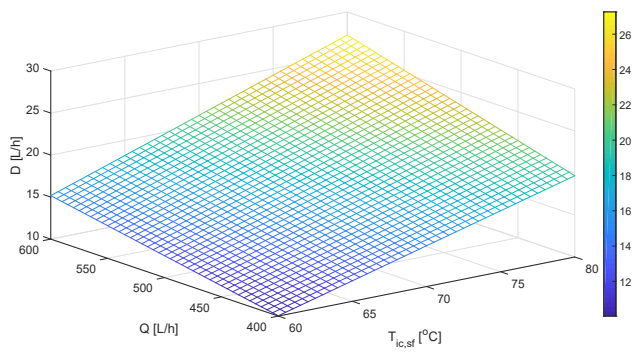

Figura 4: Gráfica 3D de la producción de destilado.

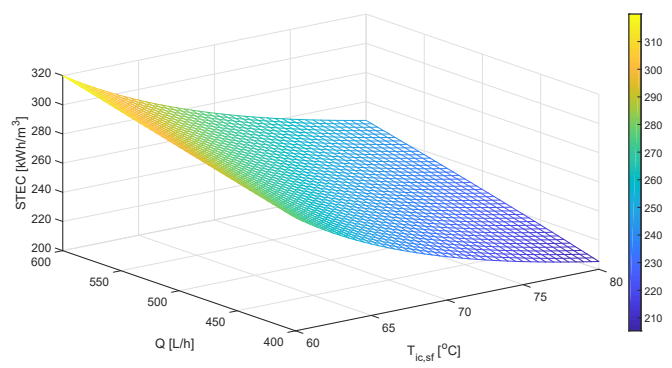

Figura 5: Gráfica 3D del STEC.

Como se puede apreciar en la Fig. 4, la máxima producción del módulo se consigue trabajando a máxima temperatura y máximo caudal. Por otro lado, basándose en la propia definición del STEC, a menor STEC, se conseguirá una operación más eficiente de la planta, ya que se requerirá menos cantidad de energía térmica para producir un volumen de destilado. De este modo, en la Fig. 5 se puede apreciar como el STEC mínimo se consigue operando a máxima temperatura y mínimo caudal. Por tanto, para minimizar el STEC y maximizar la producción de destilado, se requieren condiciones de operación en el caudal de alimentación de los módulos contrarias.

\section{Sistema de control}

En base al análisis realizado en la sección anterior, para maximizar la eficiencia térmica de la planta, se debe trabajar con el caudal de alimentación de cada módulo al mínimo. Sin embargo, el hecho de tener que cubrir una demanda de agua variable 
(consumo del invernadero), hace que la producción de los módulos tenga que aumentar en determinado momentos, y por tanto, que estos no puedan operar constantemente a caudal mínimo. Este hecho, pone de manifiesto la necesidad de desarrollar algoritmos capaces de gestionar el caudal de alimentación de los módulos, dependiendo de la demanda de agua del invernadero en cada instante, y que tengan como objetivo principal conseguir una operación eficiente de la planta de desalación. Para este propósito, se ha desarrollado un esquema de control PNMPC [13], el cual, determinará los caudales de alimentación óptimos en cada módulo $\mathrm{MD}\left(\mathrm{Q}_{1}, \mathrm{Q}_{2}, \mathrm{Q}_{3}\right.$ y $\left.\mathrm{Q}_{4}\right)$ en base a las condiciones de operación en cada momento (ver Fig. 6). Además, el algoritmo PNMPC se ha complementado con una técnica de predicción de la radiación [12], con el fin de obtener unas predicciones del comportamiento de la planta más precisas.

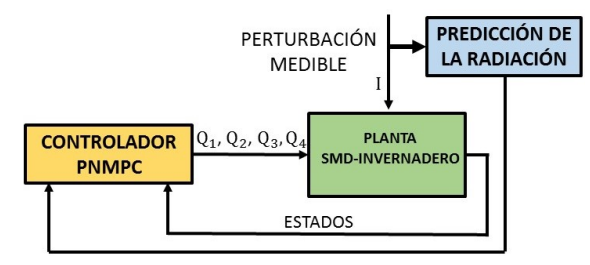

Figura 6: Esquema del controlador PNMPC

\subsection{Algoritmo PNMPC}

La técnica PNMPC se utilizará para predecir el nivel del tanque de destilado y el STEC medio de los cuatros módulos MD que componen la planta, en función de las señales de control futuras.

El algoritmo PNMPC, como todas las técnicas $\mathrm{MPC}$, se caracteriza por el uso de un vector de predicciones $\hat{\mathbf{Y}}$, el cual incluye las salidas futuras en un determinado horizonte de predicción $N$, en función de los cambios de control futuros $\Delta \mathbf{U}$ :

$$
\hat{\mathbf{Y}}=\mathbf{F}+\mathbf{G} \cdot \Delta \mathbf{U}
$$

donde $\mathbf{F}$ es la respuesta libre y $\mathbf{G} \cdot \boldsymbol{\Delta} \mathbf{U}$ es la respuesta forzada. En los algoritmos MPC clásicos, $\hat{\mathbf{Y}}$ se estima mediante el modelo lineal del sistema. Sin embargo, en la técnica PNMPC, $\hat{\mathbf{Y}}$ se calcula haciendo uso del modelo no lineal presentado en la Sección 3. De este modo, siguiendo el procedimiento propuesto en [13], la técnica PNMPC se usa para estimar $\mathbf{F}$ y $\mathbf{G}$ en cada instante de muestreo. La formulación de la técnica viene dada por la siguientes ecuaciones:

$$
\left[\begin{array}{c}
\hat{\mathbf{Y}}_{1} \\
\hat{\mathbf{Y}}_{2}
\end{array}\right]=\left[\begin{array}{l}
\mathbf{F}_{1} \\
\mathbf{F}_{2}
\end{array}\right]+\mathbf{G}_{\mathrm{PNMPC}} \cdot\left[\begin{array}{c}
\boldsymbol{\Delta} \mathbf{U}_{1} \\
\boldsymbol{\Delta} \mathbf{U}_{2} \\
\boldsymbol{\Delta} \mathbf{U}_{3} \\
\boldsymbol{\Delta} \mathbf{U}_{4}
\end{array}\right]
$$

donde:

$$
\begin{gathered}
\mathbf{F}_{i}=f\left(\mathbf{y}_{\mathbf{p}}, \boldsymbol{\Delta} \mathbf{u}_{\mathbf{p}}\right), \\
\mathbf{G}_{\mathrm{PNMPC}}=\left[\begin{array}{llll}
\frac{\partial \hat{\mathbf{Y}}_{\mathbf{1}}}{\partial \mathbf{U}_{1}} & \frac{\partial \hat{\mathbf{Y}}_{\mathbf{1}}}{\partial \mathbf{U}_{2}} & \frac{\partial \hat{\mathbf{Y}}_{\mathbf{1}}}{\partial \mathbf{U}_{3}} & \frac{\partial \hat{\mathbf{Y}}_{\mathbf{1}}}{\partial \mathbf{U}_{4}} \\
\frac{\partial \hat{\mathbf{Y}}_{\mathbf{2}}}{\partial \mathbf{U}_{1}} & \frac{\partial \hat{\mathbf{Y}}_{2}}{\partial \mathbf{U}_{2}} & \frac{\partial \hat{\mathbf{Y}}_{\mathbf{2}}}{\partial \mathbf{U}_{3}} & \frac{\partial \hat{\mathbf{Y}}_{\mathbf{2}}}{\partial \mathbf{U}_{4}}
\end{array}\right],
\end{gathered}
$$

$\mathbf{y}_{\mathbf{p}}$ es un conjunto de valores pasados y presentes de las salidas y $\boldsymbol{\Delta} \mathbf{u}_{\mathbf{p}}$ es un conjunto de valores pasados de las entradas. $\mathbf{Y}_{1}$ es la predicción del STEC medio de los cuatro módulos, $\mathbf{Y}_{2}$ es la predicción del nivel del tanque de destilado, y $\mathbf{U}_{1}, \mathbf{U}_{2}$, $\mathbf{U}_{3}$ y $\mathbf{U}_{4}$, son los caudales de cada módulo $\left(\mathrm{Q}_{1}\right.$, $\mathrm{Q}_{2}, \mathrm{Q}_{3}$ y $\left.\mathrm{Q}_{4}\right)$. Se debe tener en cuenta que esta técnica solo proporciona una aproximación de las predicciones, sin embargo, esta aproximación se ajusta mejor al comportamiento del sistema que un modelo lineal, ya que se utilizan modelos linealizados en cada instante de muestreo para calcular $\mathbf{G}_{\text {PNMPC }}$, mientras que el modelo no lineal de la planta se usa para calcular $\mathbf{F}$ manteniendo las futuras entradas de control contantes.

\subsubsection{Función de coste}

Como se ha mencionado anteriormente, el objetivo principal consiste en maximizar la eficiencia térmica de la planta de desalación. No obstante, también se pretende asegurar el suministro de agua demandado por el invernadero. Para este propósito, se intentará mantener el tanque de destilado en un nivel mayor o igual a $1500 \mathrm{~L}$, lo que aseguraría el abastecimiento del invernadero durante dos días, ya que el consumo medio por día es de alrededor de 750 L. Se debe hacer notar que, el mantenimiento del nivel deseado del tanque de destilado se puede incluir como una restricción no lineal en el problema de optimización. Sin embargo, este hecho, en combinación con las restricciones que se presentan en la siguiente subsección, daría lugar a soluciones infactibles del problema en días en los que la planta SMD tenga una producción más baja. Así, siguiendo las técnicas de suavizado de restricciones presentadas en [9], la restricción del nivel del tanque se incluye en la función objetivo, ponderada por un factor $\lambda$ que tomará diferentes valores en función del nivel medio del tanque en cada instante de muestreo:

$$
J=\sum_{j=1}^{N} \delta(j) \hat{Y}_{1}(k+j \mid k)+\sum_{j=1}^{N} \lambda(j)\left(1500-\hat{Y}_{2}(k+j \mid k)\right),
$$


donde $N$ es el horizonte de predicción, $\hat{Y}_{1}(k+j \mid k)$ es la predicción del STEC medio de los cuatro módulos e $\hat{Y}_{2}(k+j \mid k)$ es la predicción del nivel del tanque de destilado, ambas calculadas en el instante $k+j$ con la información adquirida en el instante de muestreo $k$. Por otra parte, $\delta(j)$ y $\lambda(j)$ son factores de ponderación que varían su valor en función del nivel del tanque de destilado en cada instante de muestreo. Para ello, se comprueba si el nivel medio del tanque durante los diez minutos anteriores al muestreo de la técnica PNMPC es mayor o menor que 1500 L. En el primer caso, los valores de $\delta(j)$ y $\lambda(j)$ serán de 0.001 y 1 respectivamente, en el segundo contrario $\delta(j)=1$ mientras que $\lambda(j)=0$. De este modo, si el nivel del tanque no alcanza el nivel deseado, en la función objetivo se tendrá en cuenta la restricción del nivel, por el contrario, si el nivel del tanque supera el nivel deseado, la función objetivo tendrá como objetivo único minimizar el STEC.

Para resolver el problema de optimización se ha utilizado la función fmincon de MATLAB. Este método está basado en el algoritmo del punto interior, y ha sido seleccionado ya que proporciona buenos resultados (con bajos requerimientos de tiempo computacional) en problemas como el presentado en este trabajo, en los cuales, el sistema tiene un comportamiento no lineal suave y el problema está sujeto a restricciones lineales (ver siguiente subsección).

\subsubsection{Restricciones}

En primer lugar, se ha limitado a $50 \mathrm{~L} / \mathrm{h}$ el incremento máximo y mínimo permitido en las señales de control en cada instante de muestreo (slew rate). Esta restricción está definida en la Ec. 16, y se ha impuesto ya que al trabajar con módulos iguales, se pueden llegar a múltiples soluciones óptimas, lo que puede provocar continuos saltos de caudal en los diferentes módulos.

$$
\begin{aligned}
& {\left[\boldsymbol{\Delta} \mathbf{U}_{\mathbf{i}_{\text {min }}}\right] \leq\left[\boldsymbol{\Delta} \mathbf{U}_{\mathbf{i}}(\mathrm{k}+\mathrm{j} \mid \mathrm{k})\right] \leq\left[\boldsymbol{\Delta} \mathbf{U}_{\mathbf{i}_{\max }}\right]} \\
& j=0, \ldots, N_{u}-1 \text {, }
\end{aligned}
$$

donde $N u$ es el horizonte de control.

La segunda restricción, Eq. 17, limita el caudal de funcionamiento máximo y mínimo permitido por cada módulo MD (400-600 L/h).

$$
\begin{gathered}
{\left[\mathbf{U}_{\mathbf{i}_{\text {min }}}\right] \leq\left[\mathbf{U}_{\mathbf{i}}(\mathrm{k}+\mathrm{j} \mid \mathrm{k})\right] \leq\left[\mathbf{U}_{\mathbf{i}_{\max }}\right]} \\
j=0, \ldots, N_{u}-1 .
\end{gathered}
$$

Finalmente, también se han introducido como restricciones el nivel máximo del tanque de alimentación $(3000$ L) y el rango de funcionamiento de la bomba 1 (1600-2000 L/h).

\subsection{Técnica de estimación de la radiación.}

Con el objetivo de mejorar la actuación de la estrategia PNMPC, se utiliza la técnica DES para estimar la irradiancia global, siguiendo las ideas presentadas en [12]. Esta técnica viene descrita por las siguientes ecuaciones:

$$
\begin{gathered}
S_{k}=\alpha y_{k}+(1-\alpha)\left(S_{k-1}+b_{k-1}\right), \\
b_{k}=\theta\left(S_{k}-S_{k-1}\right)+(1-\theta) b_{k-1},
\end{gathered}
$$

donde $y_{k}$ es la medida real en el instante $k$. La primera expresión proporciona el valor estimado $\left(S_{k}\right)$, mientras que la segunda proporciona la tendencia estimada $\left(b_{k}\right)$, la cual, se calcula usando valores actuales y pasados de la serie. Las contantes $\alpha$ and $\theta \in(0,1)$ han sido obtenidas mediante técnicas de optimización haciendo uso de datos de irradiancia experimentales. De esta forma, la estimación de $m$ periodos viene dada por la siguiente expresión:

$$
\hat{y}_{k+m}=S_{k}+m b_{k} .
$$

Hay diferentes formas de inicializar $S_{k}$ y $b_{k}$ [11]. En este caso, $S_{o}=y_{k}$ y $b_{o}=y_{k}-y_{k-1}$. Esta técnica ha sido implementada para estimar los $N$ futuros valores de acuerdo al horizonte de predicción del controlador PNMPC.

\subsection{Procedimiento de parada y arranque de los módulos}

Debido a que los módulos MD producen muy poco destilado cuando funcionan a una temperatura más baja de $60^{\circ} \mathrm{C}$, se ha desarrollado un procedimiento de arranque y parada de los módulos, el cual es ejecutado cada 10 minutos (de acuerdo al tiempo de muestreo de la técnica PNMPC) con el objetivo de evitar problemas de chattering. De este modo, se utiliza el modelo estático del intercambiador presentado en la Sección 3 para comprobar si la temperatura proveniente del tanque de almacenamiento permite operar los módulos a una temperatura superior a $60{ }^{\circ} \mathrm{C}$. De modo que los módulos se arrancan o se paran si se cumple dicha condición o no.

\section{Resultados}

La estrategia de control se ha probado en simulación, utilizando datos meteorológicos de la Estación Experimental de la Fundación Cajamar, del día 1 de abril de 2015. Se han llevado a cabo diferentes ensayos con un tiempo de muestreo de 10 minutos y utilizando diferentes valores del horizonte de predicción y control. Los valores de la técnica DES empleados han sido $\alpha=0.9$ y $\theta=0.98$. 

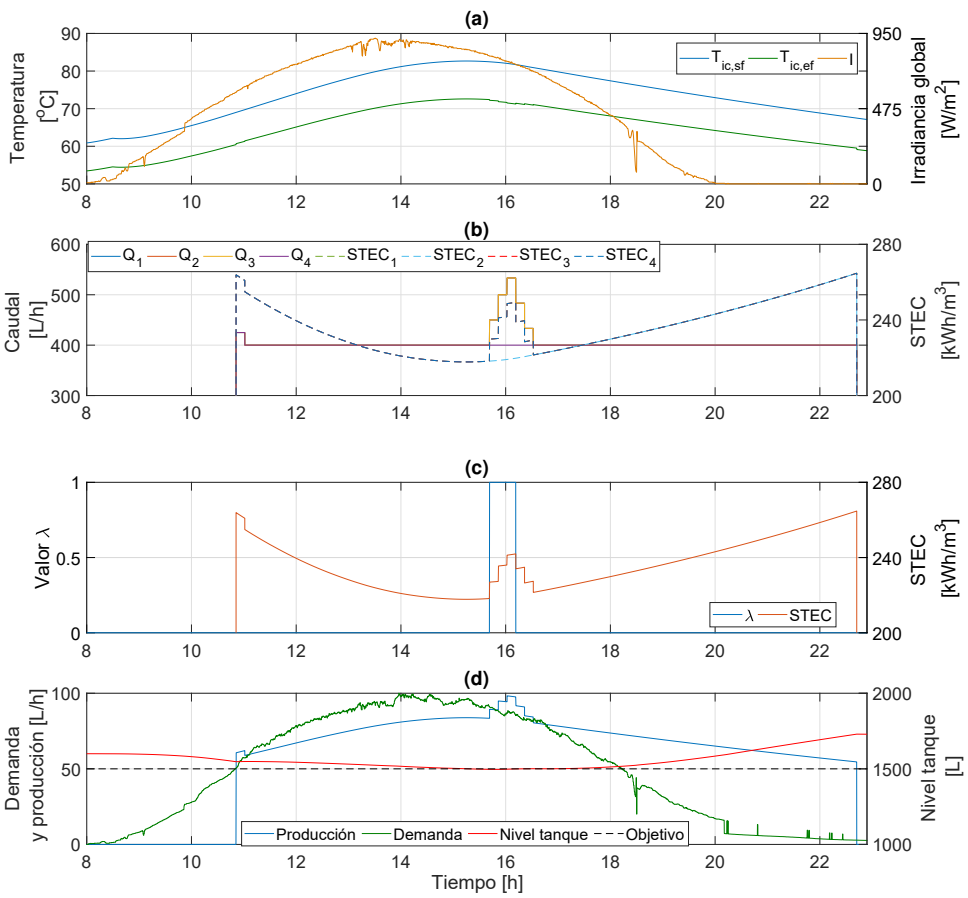

Figura 7: Resultados obtenidos en simulación con $N=N_{u}=2$.

En la Fig. 7, se muestra un ensayo llevado a cabo con $N=N_{u}=2$. En la Fig. 7a, se pueden observar las temperaturas de entrada y salida por la parte fría del intercambiador $\left(\mathrm{T}_{i c, e f}\right.$ y $\left.\mathrm{T}_{i c, s f}\right)$ y la radiación (I). En la Fig. 7b, se muestran los caudales de alimentación de cada módulo $\left(\mathrm{Q}_{1}, \mathrm{Q}_{2}, \mathrm{Q}_{3} \mathrm{y}\right.$ $\left.\mathrm{Q}_{4}\right)$ y el STEC de cada módulo $\left(\mathrm{STEC}_{1}, \mathrm{STEC}_{2}\right.$, $\mathrm{STEC}_{3}$ y $\left.\mathrm{STEC}_{4}\right)$. La Fig. 7c muestra el STEC medio de los cuatros módulos (variable a minimizar en la estrategia PNMPC) y el valor del peso que pondera a la restricción del tanque en la función objetivo $(\lambda)$. En la Fig. 7d se puede observar la producción de la planta SMD, la demanda del invernadero, el nivel del tanque de destilado, y el nivel deseado (objetivo).

Como se puede apreciar, la estrategia PNMPC mantiene los módulos operando a caudal mínimo, siempre y cuando el nivel del tanque sea superior al nivel deseado (ver Fig. 7b y d). En torno a las $15.5 \mathrm{~h}$, el nivel del tanque baja por debajo del nivel deseado (ver Fig. 7d), por lo que el valor de $\lambda$ cambia a 1 (Fig. 7c). De este modo, la estrategia PNMPC aumenta el caudal de los módulos 1, 2 y 3 , intentando mantener el nivel del tanque deseado (ver Fig. 7b y d). En la Fig. 7c, se puede observar como el STEC aumenta cuando la estrategia PNMPC incrementa los caudales de los módulos.

Para destacar las mejoras obtenidas mediante la aplicación de la técnica PNMPC, en la Tab. 1 se comparan los resultados obtenidos con una operación con los caudales fijos a $500 \mathrm{~L} / \mathrm{h}$ $\left(\mathrm{Q}_{1}=\mathrm{Q}_{2}=\mathrm{Q}_{3}=\mathrm{Q}_{4}=500 \mathrm{~L} / \mathrm{h}\right)$. Se puede observar
Tabla 1: Comparación de resultados.

\begin{tabular}{|c|c|c|}
\cline { 3 - 3 } \multicolumn{2}{c|}{} & STEC $\left[\mathrm{kWh} / \mathrm{m}^{3}\right]$ \\
\hline \multirow{4}{*}{ PNMPC } & No & 252.0955 \\
\cline { 2 - 3 } & $N=N u=1$ & 236.1308 \\
\cline { 2 - 3 } & $N=N u=2$ & 236.1309 \\
\cline { 2 - 3 } & $N=N u=3$ & 236.1309 \\
\hline
\end{tabular}

que la aplicación de la técnica PNMPC disminuye el STEC medio en unos $15.97 \mathrm{kWh} / \mathrm{m}^{3}$.

Se debe destacar que la estrategia de control desarrollada puede ser fácilmente modificada para realizar el diseño óptimo de la planta SMD en base a la demanda del invernadero.

\section{Conclusiones y trabajos futuros}

En este trabajo se ha desarrollado un algoritmo de control predictivo no lineal para la operación eficiente, en términos de eficiencia térmica, de una planta formada por un sistema de desalación SMD y un invernadero. Los resultados obtenido en simulación, muestran que es posible cubrir la demanda de agua del invernadero. Además, la operación lograda mediante la aplicación del sistema de control, requiere de media $15.97 \mathrm{kWh}$ menos de energía térmica para producir $1 \mathrm{~m}^{3}$ de destilado.

Los trabajos futuros estarán centrados en la modificación del algoritmo desarrollado para la realización de diseños óptimos de las plantas SMD, en base a una demanda de agua determinada. 


\section{Agradecimientos}

Este trabajo ha sido realizado con el apoyo del proyecto DPI2014-56364-C2-1/2-R (ENERPRO), financiado por el Ministerio de Economía, Competitividad e Industria y fondos FEDER.

\section{Referencias}

[1] A. Alkhudhiri, N. Darwish, and N. Hilal, "Membrane distillation: a comprehensive review," Desalination, vol. 287, pp. 2-18, 2012.

[2] H. Chang, S.-G. Lyu, C.-M. Tsai, Y.-H. Chen, T.-W. Cheng, and Y.-H. Chou, "Experimental and simulation study of a solar thermal driven membrane distillation desalination process," Desalination, vol. 286, pp. 400411, 2012.

[3] A. de la Calle, L. Roca, J. Bonilla, and P. Palenzuela, "Dynamic modeling and simulation of a double-effect absorption heat pump," International Journal of Refrigeration, vol. 72, pp. 171-191, 2016.

[4] M. Fernández, A. González, J. Carreño, C. Pérez, and S. Bonachela, "Analysis of on-farm irrigation performance in mediterranean greenhouses," Agricultural Water Management, vol. 89, no. 3, pp. 251-260, 2007.

[5] J. D. Gil, L. Roca, A. Ruiz-Aguirre, G. Zaragoza, J. L. Guzmán, and M. Berenguel, "Using a nonlinear model predictive control strategy for the efficient operation of a solarpowered membrane distillation system," in 25th Mediterranean Conference on Control and Automation (MED 2017). Valleta, Malta, 2017, (Aceptado).

[6] J. D. Gil, A. Ruiz-Aguirre, L. Roca, G. Zaragoza, and M. Berenguel, "Solar membrane distillation: A control perspective," in 23th Mediterranean Conference on Control and Automation (MED 2015). Torremolinos, Málaga, Spain, 2015, pp. 796-802.

[7] M. Khayet, "Solar desalination by membrane distillation: Dispersion in energy consumption analysis and water production costs (a review)," Desalination, vol. 308, pp. 89-101, 2013.

[8] M. Khayet and C. Cojocaru, "Artificial neural network modeling and optimization of desalination by air gap membrane distillation," Separation and Purification Technology, vol. 86, pp. 171-182, 2012.

[9] J. M. Maciejowski, Predictive control: with constraints. Pearson education, 2002.
[10] F. S. Martos, A. P. Bosch, and J. Calaforra, "Hydrogeochemical processes in an arid region of europe (almeria, SE Spain)," $A p$ plied Geochemistry, vol. 14, no. 6, pp. 735745, 1999.

[11] NIST. ring statistics handbook. url http://www.itl.nist.gov/div898/handbook/.

[12] A. Pawlowski, J. L. Guzmán, F. Rodríguez, M. Berenguel, and J. E. Normey-Rico, "Predictive control with disturbance forecasting for greenhouse diurnal temperature control," IFAC Proceedings Volumes, vol. 44, no. 1, pp. 1779-1784, 2011.

[13] A. Plucenio, D. Pagano, A. Bruciapaglia, and J. Normey-Rico, "A practical approach to predictive control for nonlinear processes," IFAC Proceedings Volumes, vol. 40, no. 12, pp. 210-215, 2007.

[14] R. Porrazzo, A. Cipollina, M. Galluzzo, and G. Micale, "A neural network-based optimizing control system for a seawaterdesalination solar-powered membrane distillation unit," Computers \&5 Chemical Engineering, vol. 54, pp. 79-96, 2013.

[15] M. R. Qtaishat and F. Banat, "Desalination by solar powered membrane distillation systems," Desalination, vol. 308, pp. 186-197, 2013.

[16] L. Roca, J. A. Sánchez, F. Rodríguez, J. Bonilla, A. de la Calle, and M. Berenguel, "Predictive control applied to a solar desalination plant connected to a greenhouse with daily variation of irrigation water demand," Energies, vol. 9, no. 3, p. 194, 2016.

[17] F. Rodríguez, M. Berenguel, J. L. Guzmán, and A. Ramírez-Arias, Modeling and control of greenhouse crop growth. Springer, 2015.

[18] A. Ruiz-Aguirre, D.-C. Alarcón-Padilla, and G. Zaragoza, "Productivity analysis of two spiral-wound membrane distillation prototypes coupled with solar energy," Desalination and Water Treatment, vol. 55, no. 10, pp. 2777-2785, 2015.

[19] G. Zaragoza, A. Ruiz-Aguirre, and E. Guillén-Burrieza, "Efficiency in the use of solar thermal energy of small membrane desalination systems for decentralized water production," Applied Energy, vol. 130, pp. 491-499, 2014. 